\title{
Why are Women Still Dying from Nausea and Vomiting of Pregnancy?
}

\author{
Marlena S Fejzo ${ }^{1,2 *}$, Kimber MacGibbon ${ }^{3}$ and Patrick M Mullin² \\ ${ }^{1}$ Department of Medicine, University of California, Los Angeles, Los Angeles, CA, USA \\ ${ }^{2}$ Department of Maternal-Fetal Medicine, Keck School of Medicine, University of Southern California, Los Angeles, CA, USA \\ ${ }^{3}$ HER Foundation, Damascus, OR, USA
}

${ }^{*}$ Corresponding author: Marlena S Fejzo, University of California, Los Angeles, Department of Medicine, Los Angeles, CA, USA, Tel: (310)206-1408, E-mail: mfejzo@mednet.ucla.edu

Received date: 13 May, 2016; Accepted date: 30 Jun, 2016; Published date: 4 July, 2016

Citation: Fejzo MS, Mac Gibbon K, Mullin PM. Why are women still dying from nausea and vomiting of pregnancy?, Gynecol Obstet Case Rep. 2016, 2:2.

\section{Précis}

Prompt testing and treatment followed by frequent followup with the patient until symptoms subside may help to prevent Hyperemesis Gravidarum related deaths in the future.

\section{Abstract}

Until the 1950's, maternal deaths were commonly associated with hyperemesis gravidarum (HG). Although maternal mortality secondary to HG has since decreased, there have been 6 deaths reported recently in the literature. In addition, reports of Wernicke's encephalopathy (WE) secondary to HG are on the rise. Herein we review the documented mortalities and provide our analysis of the critical issues that must be urgently addressed to put an end to HG-related deaths. In short, we recommend the following for patients with HG: 1) mandatory reporting and documentation on death certificates of HG-related deaths, 2) inclusion of appropriate thiamin dose in all prenatal vitamin formulations, 3) daily electrolyte and nutrition monitoring in patients presenting with HG with special attention to and hospitalization for signs of thiamin deficiency and hypokalemia, 4) thyroid screening and subsequent treatment for all patients presenting with $\mathrm{HG}, 5$ ) provider education on the importance of communication with the patient/family as language barriers and provider bias can result in misdiagnosing symptoms of WE when depression and/or inability to communicate is present. Prompt testing and treatment followed by frequent follow-up with the patient until symptoms subside may help to prevent HG-related deaths in the future.

Keywords: Hyperemesis gravidarum; Pregnancy; Wernicke's encephalopathy; Osmotic demyelination; Thiamin; Hypokalemia; Thyrotoxicosis; Mortality

\section{Review}

Hyperemesis Gravidarum (HG), a severe form of nausea and vomiting of pregnancy, occurs in approximately $0.3 \%-2 \%$ of pregnancies and leads to significant weight loss, dehydration, electrolyte imbalance, and ketonuria [1]. HG accounts for over 375,000 hospital discharges in the US annually and is associated with significant morbidity such as Wernicke's encephalopathy, renal failure, liver function abnormalities, and esophageal rupture [1]. HG is also associated with an increased risk of adverse outcome including low birth weight, intrauterine growth restriction, preterm delivery, fetal and neonatal death, and neurodevelopmental delay in exposed children [1]. Of particular concern, it is still a cause of maternal mortality with 6 cases of maternal death secondary to HG reported recently [1]. These deaths, briefly summarized in Table 1, were complicated with diagnoses including Wernicke's encephalopathy, osmotic demyelination syndrome, seizures, hypokalemia, thyroid storm, dehydration, and/or severe thyrotoxicosis.

Patient 1 first presented to hospital in the United States at 9 weeks gestational age with $\mathrm{HG}$ and serum biochemistry of hypokalemia, ketonuria, and hematuria. She was discharged with a diagnosis of depression and hyponatremia and prescribed ondansetron and PremesisRx (a prenatal vitamin lacking thiamin) and told to increase protein and salt intake. She returned to hospital the following day and over the course of the next few weeks was diagnosed with WE, osmotic demyelination syndrome, and seizures. It was noted that English was not her primary language. She died of respiratory failure at 14 weeks gestational age. Patient 2 presented in hospital in Tunisia with HG at 20 weeks gestational age with serum biochemistry of hypokalemia. She was diagnosed with WE and treated with $900 \mathrm{mg}$ parenteral thiamin daily. She died of cardiorespiratory arrest. The authors of reports on these 2 patients conclude that prompt treatment with fluid, parenteral vitamins, nutritional support, and methodical electrolyte replacement with daily monitoring can prevent HG-related deaths. The third patient presented in a hospital in Oman with HG at 22 weeks with a serum biochemistry of hypokalemia, hyper creatinine, hypophosphate, and low serum albumin. She was diagnosed with WE followed by central pontine 
myelinolysis. She was treated with $100 \mathrm{mg}$ parenteral thiamin 3 times daily and vitamins, electrolytes, and trace elements. She died of multi-organ failure at 30 weeks gestation. The authors report that CPM likely occurred due to hypokalemia, hypernatremia, and hyperosmolality in HG. Cases 4-6 were reported in the first program ever worldwide to implement confidential enquiries into maternal deaths.

Table 1 Summary of 6 maternal mortalities secondary to HG

\begin{tabular}{|c|c|c|c|c|c|}
\hline Cause of death & Origin of death & $\begin{array}{l}\text { Serum biochemistry on } \\
\text { presentation }\end{array}$ & Diagnosis & $\begin{array}{l}\text { Gestational Age } \\
\text { Presented/Death }\end{array}$ & Reference \\
\hline Respiratory Failure & United States & $\begin{array}{l}\text { Hypokalemia, ketonuria, } \\
\text { hematuria }\end{array}$ & $\begin{array}{l}\text { WE, osmotic } \\
\text { demyelination } \\
\text { syndrome, seizures }\end{array}$ & 9 wks $/ 14 w_{k s}{ }^{\dagger}$ & $\begin{array}{l}\text { MacGibbon et al. } \\
{[1]}\end{array}$ \\
\hline Cardiorespiratory Arrest & Tunisia & hypokalemia & WE§ & $20 w k s / N A$ & Walid et al. 2012 \\
\hline Multiorgan failure & Oman & $\begin{array}{l}\text { hypokalemia, hyper } \\
\text { creatinine, hypophosphate, } \\
\text { low albumin }\end{array}$ & $\begin{array}{l}\text { WE, central pontine } \\
\text { myelinolysis }\end{array}$ & 22 wks/30 wks & Kantor et al. 2014 \\
\hline $\begin{array}{l}\text { Thyroid storm or } \\
\text { hypokalemia }\end{array}$ & UK/Ireland ${ }^{\ddagger}$ & $\begin{array}{l}\text { hypokalemia, } \\
\text { thyrotoxicosis, tachycardia }\end{array}$ & $\begin{array}{l}\text { hypokalemia, } \\
\text { thyrotoxicosis }\end{array}$ & NA/NA* ${ }^{*}$ & Knight et al. [5] \\
\hline Severe thyrotoxicosis & UK/Ireland & thyrotoxicosis & thyrotoxicosis & NA/NA & Knight et al. [5] \\
\hline $\begin{array}{l}\text { Sudden Unexplained Death } \\
\text { in Epilepsy }\end{array}$ & UK/Ireland & hypokalemia & seizures & NA/NA & Knight et al. [5] \\
\hline
\end{tabular}

The report identified $3 \mathrm{HG}$-related deaths occurring in the UK/Ireland between 2009-2012. The 4th case presented with hypokalemia, thyrotoxicosis, and tachycardia, but was only treated for thyrotoxicosis, and not antiemetics nor potassium replacement. The cause of death was determined to be from either thyroid storm and/or hypokalemia. The 5th case presented with and apparently died from thyrotoxicosis, but was noted to have "exemplary treatment." This illustrates the difficulty in treating patients with HG accompanied by severe thyroid abnormalities, and suggests early screening and prompt treatment for thyroid abnormalities in all cases of severe and prolonged nausea/vomiting of pregnancy may be warranted. The 6th mortality presented with hypokalemia with a diagnosis of seizures and sudden unexplained death in epilepsy. The authors report that this woman was likely unable to ingest drugs due to vomiting, but was placed in a single room and not seen by a doctor for all 3 days prior to her death. This case illustrates the need for consistent supervision and communication with women suffering from HG. In reviewing these cases, it is clear that by implementation of 5 important steps we can limit future deaths secondary to HG. These steps are outlined below.

\section{Mandate record-keeping}

HG was a common cause of maternal mortality until the 1950s when intravenous hydration was introduced. It is believed that English novelist and poet Charlotte Brontë died in 1855 of early pregnancy complications secondary to HG, as she wrote shortly before her death early in pregnancy in 1855 , "my sufferings are very great-my nights indescribable -sickness with scarce a reprieve-I strain until what I vomit is mixed with blood." However her death certificate reports phthisis as cause of death with no mention of her self-described malnourishment due to nausea and vomiting in early pregnancy [2]. Interestingly, report of a death due to HG in the United States in 1908 reports "Cause of death: Exhaustion from excessive vomiting. Contributory: Pregnancy" [3]. Yet in Brontë's case, and in cases today, there is, as far as we know, no mention of pregnancy or hyperemesis gravidarum in primary maternal death reports. For example, the recent death of a US patient was coded as "respiratory failure", and the autopsy listed her cause of death as "diffuse leukoencephalopathy," thus obscuring the diagnosis of HG [1].

In addition, Creanga et al. recently reported that maternal deaths are on the rise, with 3,358 pregnancy-related deaths in the US between 2006-2010 [4]. Approximately 20\% of those are undelivered and potentially related to HG. However, it is impossible to determine the number of deaths secondary to HG because vital statistics may report cardiac arrest or respiratory failure with no mention of the primary cause. The UK has implemented a confidential inquiry into maternal deaths while in the US and other countries HG-related deaths are undocumented [5]. We recommend mandating coding for HG on hospital cause of death records and death certificates. Or, at a minimum, with electronic medical records there should be a clear path to cross-linking maternal deaths with HG diagnoses. In the meantime, we recommend all HG-related deaths in the US be reported confidentially to the Hyperemesis Education and Research Foundation (www.hyperemesis.org) in an effort to document, investigate, and prevent future deaths.

\section{Inclusion of an appropriate thiamin dose in prenatal vitamins}

Three out of the 6 recently reported deaths were complicated by Wernicke's encephalopathydue to thiamin deficiency. Reports of WE secondary to HG are on the rise, with over 25 cases published between 2012-2015 [1]. This may 
be due to the trend toward emergency department treat and release and/or outpatient treatment for HG [6]. Thiamin is not included in some prenatal formulations and may be included at sub-standard levels in others. In addition, many women who develop HG are unable to tolerate their prenatal vitamins. To prevent the development of thiamine deficiency, a prenatal vitamin with a minimum of $5 \mathrm{mg}$ of thiamine is recommended. If the prenatal vitamin is not tolerated daily, the patient should be instructed to try taking a supplement with only thiamin in doses exceeding $5 \mathrm{mg}$, or one with limited ingredients such as just thiamin and pyridoxine. If these are not tolerated, intravenous thiamin is recommended. Because thiamin is depleted rapidly in early pregnancy and again in late pregnancy, when thiamin demands are on the rise, women with prolonged symptoms and/or weight loss who are unable to tolerate prenatal vitamins that include thiamin, should have thiamin levels followed regularly. Basic screening for signs of WE should be shared with the patient and her family/ caretakers including signs of ataxia, confusion, and oculomotor symptoms, which may be described by the patient or partner/ caretaker as apathy, weakness, speech and gait abnormalities, and lack of focus [1]. It should be remembered that thiamin deficiency mimics HG, making it difficult at times to determine when thiamin deficiency is present. Given thiamin is non-toxic, giving thiamin IV proactively is strongly advisable.

\section{Electrolyte monitoring and treatment}

Potassium deficiency was first reported in 1953 in association with 8 cases of severe HG all accompanied by lethargy, listlessness, apathy, mental depression, and muscle weakness [7]. In 1953 it was noted that therapy with intravenous potassium chloride resulted in notable improvement in mental attitude and muscular strength and appeared to be "a lifesaving procedure". Five out of 6 of the recent patients who died from complications secondary to $H G$, presented with hypokalemia. Therefore, patients who present with HG accompanied by hypokalemia may represent a highrisk subgroup that should be hospitalized, closely monitored for electrolyte disturbances, and treated until complete and prolonged stabilization of potassium and other electrolyte levels is achieved.

\section{Thyrotoxicosis screening and treatment}

Hyperthyroidism accompanies HG in as many as $66 \%$ of pregnancies, yet thyroid screening and treatment of HG patients is not routine practice. 8 Mutations in the thyrotropin receptor have been linked to $H G$, suggesting this pathway may be causal in some cases $[8,9]$. Two of the 6 recent mortalities were accompanied by severe thyrotoxicosis/thyroid storm. Of note, in 1980 a patient presenting with severe thyrotoxicosis and hyperemesis gravidarum reportedly responded dramatically to Propranolol (Inderal) treatment, with return of severe vomiting upon discontinuation of Propranolol and subsequent cessation of vomiting upon restarting medication [10]. The link between thyroid disease and HG warrants further study. At a minimum, all patients presenting with HG should be screened for hyperthyroidism and treated if tests reveal abnormalities.

\section{Frequent communication and understanding}

In 1992 it was reported that patients with "HG garner little attention and engender little sympathy from their physicians" [11]. In fact, in one of the recent HG-related deaths it was reported that "the woman (hospitalized) with hyperemesis gravidarum did not see a senior doctor for her entire 5 day admission, and worse, did not see any doctor for the three days prior to her death." Additionally, in another maternal death and a recent non-fatal respiratory arrest secondary to HG, miscommunication due to language differences potentially contributed to a delayed understanding of the severity of disease, until it was too late $[1,12]$. As addressed previously, communication difficulties can be an early sign of $W E$, and should not be ignored or brushed aside as signs of depression. In addition, two of the mortalities were accompanied by seizures, which can easily be missed if the patient is not monitored closely. Understanding that HG can be fatal, it is critical that caretakers address cases of HG immediately and communicate frequently with the patient. If the patient is non-communicative or if there is a language difference, it is imperative that a family member/caretaker and/or translator be present to allow communication with a trained medical professional on a daily basis until the patient's condition improves.

It is our fear that with the trend toward a decrease in hospitalization for $\mathrm{HG}$ and an increase in outpatient and emergency department management of symptoms, there may be an increase in HG-related complications and deaths in the future. This article summarizes the steps we can take to limit future mortalities secondary to HG, which includes improving record-keeping, ensuring appropriate thiamin intake, monitoring and replenishing electrolytes, screening and treating for thyroid abnormalities, and maintaining a clear line of communication with the HG patient until their symptoms improve. While we have a long way to go to put an end to the "indescribable suffering" of severe nausea and vomiting of pregnancy described by Charlotte Brontë before her death, with these steps, it is our hope that we can at least put an end to maternal mortality associated with hyperemesis gravidarum.

\section{References}

1. MacGibbon KW, Fejzo MS, Mullin PM (2015) Mortality Secondary to Hyperemesis Gravidarum: A Case Report. Womens Health Gynecol 6(2).

2. Brontë C, Smith M (2004) The Letters of Charlotte Brontë: 1852-1855. Oxford University Press.

3. State Board of Health file No. 532530, State of Utah-Death Certificate.

4. Creanga AA, Berg CJ, Ko JY, Farr SL, Tong VT, et al. (2014) Maternal mortality and morbidity in the United States: where are we now?. J Womens Health 23(1): 3-9. 
5. Knight M, Kenyon S, Brocklehurst P, Neilson J, Shakespeare J, et al. (2014) Eds on behalf of MBRRACE- UK. Saving Lives, Improving Mothers' Care - Lessons learned to inform future maternity care from the UK and Ireland Confidential Enquiries into Maternal Deaths and Morbidity 2009-12. Oxford: National Perinatal Epidemiology Unit, University of Oxford 2014.

6. Ramzan A, Fejzo MS, Mullin P (2012)Hyperemesis gravidarum related hospitalizations and emergency room visits: characterizations and trends, 2000-2009. Am J Obstet Gynecol 206(1): S246-S247.

7. Lans HS, Gollin HA, Daro AF Nora E (1953) Hypokalemia due to persistent vomiting during pregnancy. JAMA 153(11): 1012-1015.

8. Goodwin TM, Montoro M, Mestman JH (1992) Transient hyperthyroidism and hyperemesis gravidarum: clinical aspects. Am J Obstet Gynecol 167(3): 648-52.
9. Coulon AL, Savagner F, Briet C, Vernin M, Munier M, et al. (2016) Prolonged and Severe Gestational Thyrotoxicosis Due to Enhanced hCG Sensitivity of a Mutant Thyrotropin Receptor. J Clin Endocrinol Metab 101(1): 10-1.

10. Valentine BH, Jones C, Tyack AJ (1980) Hyperemesis Gravidarum due to thyrotoxicosis. Postgraduate Medical Journal 56;746-747.

11. Abell TL, Riely CA (1992) Hyperemesis gravidarum. Gastroenterol Clin North Am 21: 835-49.

12. Iwashita A, Baba Y, Usui R, Ohkuchi A, Muto S, et al. (2015) Respiratory Arrest in an Obese Pregnant Woman with Hyperemesis Gravidarum. Case Rep Obstet Gynecol. 